\title{
General solution to two-dimensional nonslipping JKR model with a pulling force in an arbitrary direction
}

\author{
Shaohua Chen*, Tzuchiang Wang \\ Biomechanics Group, LNM, Institute of Mechanics, Chinese Academy of Sciences, Beijing 100080, People's Republic of China
}

Received 22 May 2006; accepted 13 June 2006

Available online 15 June 2006

\begin{abstract}
In this paper, a generalized JKR model is investigated, in which an elastic cylinder adhesively contacts with an elastic half space and the contact region is assumed to be perfect bonding. An external pulling force is acted on the cylinder in an arbitrary direction. The contact area changes during the pull-off process, which can be predicted using the dynamic Griffith energy balance criterion as the contact edge shifts. Full coupled solution with an oscillatory singularity is obtained and analyzed by numerical calculations. The effect of Dundurs' parameter on the pull-off process is analyzed, which shows that a nonoscillatory solution can approximate the general one under some conditions, i.e., larger pulling angle ( $\pi / 2$ is the maximum value), smaller $a / R$ or larger nondimensional parameter value of $\Delta \gamma / E^{*} R$. Relations among the contact half width, the external pulling force and the pulling angle are used to determine the pull-off force and pull-off contact half width explicitly. All the results in the present paper as basic solutions are helpful and applicable for experimenters and engineers.
\end{abstract}

(C) 2006 Elsevier Inc. All rights reserved.

Keywords: Generalized JKR model; Adhesion; Perfect bonding interface; Contact mechanics; Arbitrary direction; Basic solution

\section{Introduction}

Contact mechanics starts from Hertz theory in 1882 [1], in which two elastic bodies are pressed to contact each other by external loading without considering adhesion, i.e., molecule interactions between two adjacent bodies. However, as the contact size decreases, adhesion between the bodies becomes significant, especially for clean surfaces and lightly loaded systems. This aspect of contact mechanics was successfully studied by JKR theory in 1971 [2]. The stress field near the contact edge in JKR model has a crack-like singularity and the size of the contact region can be determined by the Griffith energy equilibrium criterion, which is larger than the contact size predicted by Hertz theory. The pull-off force in the classical JKR theory is found to be $F=3 \pi R \Delta \gamma / 2$, where $R$ is a combined radius that can be expressed by the radii of two spheres $R_{1}, R_{2}$ as $1 / R=1 / R_{1}+1 / R_{2} ; \Delta \gamma$ is the work of adhesion. Here we can

\footnotetext{
* Corresponding author. Fax: +861062561284 .

E-mail address: chenshaohua72@hotmail.com (S. Chen).
}

find that the pull-off force is independent of the elastic properties of the solids.

In JKR model, the adhesion force outside the contact region is not considered. In contrast, Derjaguin et al. [3] considered the adhesive interactions and developed the classical DMT theory model, but it assumes the same Hertz stress distribution inside the contact area. The pull-off force is found to be $F=$ $2 \pi R \Delta \gamma$.

It seems that JKR and DMT theories are competitive, which is explained by Tabor [4] that the JKR theory is especially applicable for contact between relatively large and soft elastic bodies and the DMT theory is more appropriate for contact between small and rigid particles. A unified theory (MD) linking JKR and DMT models are proposed in 1992 by [5] by extending the Dugdale model of a plastically yielded crack to adhesive contact mechanics and the crack-like singularity is eliminated to keep the continuity of stresses at the contact edge.

After development of these three famous adhesive contact theories (JKR, DMT, and MD), numerous extensions have been developed for elastic and viscoelastic bodies, for examples [6-20], in which it should be mentioned that the two- 
dimensional classical JKR and MD models have been studied by [8] and [12], respectively.

Most of the existing models on contact mechanics neglect the tangential tractions or regard the tangential stress as frictional one to uncouple the normal and tangential stresses in the contact region [21]. Kendall [22] investigated the effects of shrinkage stress on a brittle interfacial failure of a bonded laminate. Savkoor and Briggs [23] showed that an applied tangential force can reduce the area of contact between elastic solids. With the developing of bio-mechanics and bionics, especially for the gecko and some insects' adhesion and cell adhesion, contact mechanics is recently used to explain the bio-phenomena and find the mechanical mechanism in biology. As we know, the effects of tangential traction in the adhesive contact region on the pull-off process is negligible when the external force is normal to the contact interface [21], which results in many investigations of bio-adhesion using directly the classical JKR model. For example, Chu et al. [24] showed that the JKR theory can help predicting the adhesion energy between two S180 cells; More applications and extensions of adhesive contact mechanics are relative to the adhesion abilities of gecko and insects [25-34]. However, the effects of tangential stress cannot be ignored sometimes, for examples, as cells are pulled by a micro-pipette aspiration at a slant to measure the adhesion force or geckos (insects) climb on a vertical wall, tangential traction in the contact regions will work. Otherwise, cells will slip and geckos (insects) cannot stay on the vertical wall. Furthermore, recent studies on elastic bodies in nonslipping adhesive contact with a laterally stretched substrate $[35,36]$ indicate that the substrate strain can have significant effect on the contact area. The pull-off process of two elastic spheres in nonslipping adhesive contact under a pair of pulling forces and a mismatch strain will be influenced by the mismatch strain significantly [37].

Inspired by cells pulled by a micro-pipette aspiration obliquely and insects staying on vertical surfaces, the nonslipping adhesion of an elastic cylinder in contact with an elastic half space is investigated in the present paper with a pulling force in an arbitrary direction. Both solids are isotropic materials in the present study. In contrast to the classical JKR model, both the normal and tangential tractions in the contact region should be considered and the focus of the study is on how the pulling angle influences the pull-off contact width and the pull-off force comparing to the classical JKR theory. Viscoelastic material to simulate cells and anisotropic bodies to mimic geckos' (insects') feet structures are involved in future studies. The results in the present paper as basic solutions can be useful for engineering applications.

\section{Model}

The plane strain adhesive contact model is shown in Fig. 1, in which an elastic cylinder contacts with an elastic half space. The contact region is assumed to be perfect bonding, where not only the normal traction but also the tangential one can transfer across the contact interface. An external force $F$ acts on the cylinder in an angle $\theta$ to pull-off the adhesive contact as

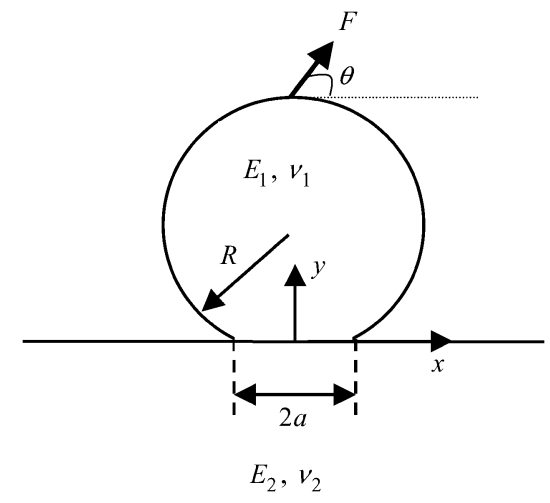

Fig. 1. Plane strain model of an elastic cylinder adhesively contacting with an elastic half plane. $F$ is an external pulling force and $\theta$ is the pulling angle. $\left(E_{1}, v_{1}\right),\left(E_{2}, v_{2}\right)$ are the Young's moduli and Poisson ratios of the cylinder and the half plane, respectively. $R$ is the radius of the cylinder. $2 a$ is the contact width.

shown in Fig. 1. $\left(E_{1}, v_{1}\right)$ and $\left(E_{2}, v_{2}\right)$ are the Young's moduli and Poisson ratios of the upper cylinder and lower half space, respectively. $R$ is the radius of the elastic cylinder. The contact half width is denoted as $a$, which can be determined by the dynamic Griffith energy balance criterion as the contact edge shifts during the pull-off process. As in almost all contact mechanics theories [21], the contact width is assumed to be small compared to the radius of the cylinder such that the deformation of the cylinder can be approximated by that of an elastic half space.

Two Cartesian reference coordinate systems $\left(x, y_{1}\right)$ and $\left(x, y_{2}\right)$ lie at the center of the contact region with $y_{1}$ and $y_{2}$ pointing into the upper cylinder and the lower half space, respectively. The normal and tangential tractions along the contact interface of the cylinder inside the contact area will be denoted as $P(x)$ and $Q(x)$. The edges of the contact region resemble two opposing interfacial cracks under plane strain deformation.

\section{Full coupled solution to the bimaterial contact model}

As mentioned in the introduction that in most of the theories, the contact interface only transfers the normal traction, while the tangential traction is neglected or looked as a friction traction related with the normal traction. In the present model, the interface is looked as a prefect bonding, which allows the transition of both the normal and tangential tractions.

Due to a much smaller contact width in contrast to the cylinder radius, the external force can be assumed to act at a remote point and the tangential force introduces shear tractions which cause a tangential shift $\omega$ of points remote from the contact area. The contact area moves in the direction of the tangential force without distortion of shape or size. This means that, if the interface is not bonded, the two solids would undergo a relative tangential displacement $\omega$. However, since the interface is bonded, this relative displacement cannot occur and is instead compensated by elastic deformation in the two solids, which causes tangential tractions in the contact area. Here, one can see that the physical picture for the model in Fig. 1 is very similar to an interface crack model, in which two half-infinite edge 
cracks between two dissimilar half spaces are subjected to remote normal and tangential forces. In this case, the continuity condition of displacements across the contact interface can be expressed as

$$
\left\{\begin{array}{l}
\bar{u}_{x 1}-\bar{u}_{x 2}=\omega, \\
\bar{u}_{y 1}+\bar{u}_{y 2}=\delta-\frac{x^{2}}{2 R}, \quad|x| \leqslant a,
\end{array}\right.
$$

where $\bar{u}_{x i}(i=1,2)$ denotes the tangential displacements on the surface of each body along the contact line and $\bar{u}_{y i}(i=$ $1,2)$ is the corresponding surface normal displacements. $\delta$ is the relative displacement of the centers of the cylinder and the half space during contact formation in $y$ direction, which has the same definition as [21] and the second term at the right hand in the second equation adopts the parabolic shape assumption of the upper cylinder.

Using the relative displacement gradients with respect to $x$

$$
\left\{\begin{array}{l}
\frac{\partial \bar{u}_{x 1}}{\partial x}-\frac{\partial \bar{u}_{x 2}}{\partial x}=0 \\
\frac{\partial \bar{u}_{y 1}}{\partial x}+\frac{\partial \bar{u}_{y 2}}{\partial x}=-\frac{x}{R},
\end{array}\right.
$$

and combining the Green functions of an elastic half space subjected to both normal and tangential tractions yields two integral equations,

$$
\left\{\begin{array}{l}
\frac{1}{\pi} \int_{-a}^{a} \frac{Q(s)}{s-x} \mathrm{~d} s-\beta P(x)=0, \\
\frac{1}{\pi} \int_{-a}^{a} \frac{P(s)}{s-x} \mathrm{~d} s+\beta Q(x)=-\frac{E^{*} x}{2 R},
\end{array}\right.
$$

where $Q(x)$ and $P(x)$ are coupled, the effective modulus $E^{*}$ is

$$
\frac{1}{E^{*}}=\frac{1-v_{1}^{2}}{E_{1}}+\frac{1-v_{2}^{2}}{E_{2}},
$$

and

$$
\beta=\frac{1}{2}\left\{\frac{\left(1-2 v_{1}\right) / \mu_{1}-\left(1-2 v_{2}\right) / \mu_{2}}{\left(1-v_{1}\right) / \mu_{1}+\left(1-v_{2}\right) / \mu_{2}}\right\}
$$

is one of the Dundurs' parameter [38]. $\mu_{1}$ and $\mu_{2}$ are shear moduli of each material.

Following a similar analysis in [35], we rewrite Eq. (3) in a matrix form

$$
\frac{1}{\pi} \int_{-a}^{a} \frac{\mathbf{A}}{s-x} \mathbf{f}(s) \mathrm{d} s+\mathbf{B f}(x)=\mathbf{C}
$$

where the vectors can be expressed as

$$
\begin{array}{ll}
\mathbf{f}(s)=\left(\begin{array}{c}
Q(s) \\
P(s)
\end{array}\right), & \mathbf{A}=\left[\begin{array}{ll}
1 & 0 \\
0 & 1
\end{array}\right]=\mathbf{I}, \\
\mathbf{B}=\left[\begin{array}{cc}
0 & -\beta \\
\beta & 0
\end{array}\right], & \mathbf{C}=\left[\begin{array}{c}
0 \\
\frac{-E^{*} x}{2 R}
\end{array}\right] .
\end{array}
$$

Introducing the following transformation:

$$
F_{k}(z)=\frac{1}{2 \pi i} \int_{-a}^{a} \frac{f_{k}(s)}{s-z} \mathrm{~d} s, \quad k=1,2,
$$

where $z=x+i y$ and $i=\sqrt{-1}$. Equation (6) can be transferred to two inhomogeneous Hilbert equations. Solving the Hilbert equations and combining the boundary conditions

$$
\int_{-a}^{a} P(x) \mathrm{d} x=-F \sin \theta, \quad \int_{-a}^{a} Q(x) \mathrm{d} x=-F \cos \theta
$$

yields the solutions to the interfacial tractions. The whole solving process is standard but very complex. Similar method has been used in [35], so that we skip all the details here and present the final interfacial tractions in the contact region,

$$
\left\{\begin{aligned}
Q(x)= & 2 \operatorname{Re}\{I(x)\}+\frac{E^{*} x \beta}{2 R\left(1-\beta^{2}\right)} \\
& -\operatorname{Im}\left\{\frac{(F \sin \theta+i F \cos \theta)(a+x)^{-\bar{r}}(a-x)^{-r}}{\pi}\right\}, \\
P(x)= & -2 \operatorname{Im}\{I(x)\} \\
& -\operatorname{Re}\left\{\frac{(F \sin \theta+i F \cos \theta)(a+x)^{-\bar{r}}(a-x)^{-r}}{\pi}\right\},
\end{aligned}\right.
$$

where

$I(x)=\frac{-i E^{*}(a+x)^{-\bar{r}}(a-x)^{-r}}{4 \pi R\left(1-\beta^{2}\right)}\left[\int_{-a}^{a} \frac{t(a+t)^{\bar{r}}(a-t)^{r}}{t-x} \mathrm{~d} t\right]$,

and $r$ is the stress singularity with an oscillatory index $\kappa$

$r=\frac{1}{2}+i \kappa, \quad \kappa=\frac{1}{2 \pi} \ln \frac{1+\beta}{1-\beta}$.

From Eq. (10), one can see that the tractions in the contact region are singular, which is very similar to that of interface crack model in fracture mechanics.

Introducing a complex-valued stress intensity factor

$K=-\sqrt{2 \pi} \lim _{x \rightarrow a}(a-x)^{r}[P(x)+i Q(x)]$,

which by Eq. (10) leads to

$$
\begin{aligned}
K= & \frac{E^{*}(2 a)^{-\bar{r}}}{\sqrt{2 \pi}\left(1-\beta^{2}\right) R} \int_{-a}^{a} \frac{t(a+t)^{\bar{r}}(a-t)^{r}}{a-t} \mathrm{~d} t \\
& +\frac{\sqrt{2}(2 a)^{-\bar{r}}(i F \cos \theta+F \sin \theta)}{\sqrt{\pi}} .
\end{aligned}
$$

Substituting Eq. (14) into the dynamic Griffith energy balance criterion

$G=\frac{1}{\cosh ^{2} \pi \kappa} \frac{|K|^{2}}{2 E^{*}}=\Delta \gamma$,

yields the final controlling equations 


$$
\begin{aligned}
& \mid \frac{1}{2\left(1-\beta^{2}\right)}\left(\frac{a}{R}\right)^{3 / 2} \int_{-1}^{1} \frac{\xi(1+\xi)^{\bar{r}}(1-\xi)^{r}}{1-\xi} \mathrm{d} \xi \\
& \quad+\left.\left(\frac{F \sin \theta}{E^{*} R}+\frac{i F \cos \theta}{E^{*} R}\right)\left(\frac{R}{a}\right)^{1 / 2}\right|^{2}=\frac{2 \pi \Delta \gamma \cosh ^{2}(\pi \kappa)}{E^{*} R},
\end{aligned}
$$

which denotes the relations among the contact half width $a$, the pulling force $F$ and the pulling angle $\theta ; \Delta \gamma$ is the work of adhesion of the contact interface, $\Delta \gamma=\gamma_{1}+\gamma_{2}-2 \gamma_{12}$, where $\gamma_{1}, \gamma_{2}$ are the intrinsic surface energies of the two solids and $\gamma_{12}$ is the surface energy of the contact interface.

From Eqs. (13)-(16), one can see that the concepts of complex-valued stress intensity factor and the Griffith energy criterion in fracture mechanics are used to describe the adhesive contact pull-off process. As the energy release rate exceeds the work of adhesion of the contact interface, debonding will happen.

\section{Nonoscillatory solution to the bimaterial contact model}

Motivated by recent studies on an elastic cylinder adhering with a stretched substrate $[35,36]$ and two adhering spheres subjected to combining actions of a pair of forces and a mismatch strain [37], in which it is found that the effects of Dundurs' parameter on the contact area and pull-off process are very small and can be neglected, a nonoscillatory solution can be a very good approximation to the bimaterial adhesive contact model, we present the corresponding nonoscillatory solution to the present model in this section.

Due to the elastic mismatch, oscillatory index $\kappa$ is introduced in Section 3, which is only related with Dundurs' parameter $\beta$. The nonoscillatory case corresponds to $\beta=0$, which yields

$r=\frac{1}{2}, \quad \kappa=0$,

then, using a similar method in [35] or substituting Eq. (17) into Eq. (10), the tangential and normal tractions of the nonoscillatory case in the contact region can be written as

$$
\begin{aligned}
& P(x)=\frac{-E^{*}}{2 R} \frac{x^{2}-a^{2} / 2}{\sqrt{a^{2}-x^{2}}}-\frac{F \sin \theta}{\pi \sqrt{a^{2}-x^{2}}}, \\
& Q(x)=\frac{-F \cos \theta}{\pi \sqrt{a^{2}-x^{2}}},
\end{aligned}
$$

which results in the uncoupled stress intensity factors as

$$
\begin{aligned}
K_{\mathrm{I}} & =-\sqrt{2 \pi} \lim _{x \rightarrow a}(a-x)^{1 / 2} P(x) \\
& =\frac{E^{*} \sqrt{\pi} a^{3 / 2}}{4 R}+\frac{F \sin \theta}{\sqrt{\pi a}}, \\
K_{\mathrm{II}} & =-\sqrt{2 \pi} \lim _{x \rightarrow a}(a-x)^{1 / 2} Q(x)=\frac{F \cos \theta}{\sqrt{\pi a}} .
\end{aligned}
$$

Substituting Eqs. (20) and (21) into the Griffith energy balance criterion for the nonoscillatory case

$\frac{\left(K_{\mathrm{I}}^{2}+K_{\mathrm{II}}^{2}\right)}{2 E^{*}}=\Delta \gamma$ yields

$$
\frac{F^{2}}{\pi a}+\frac{F \sin \theta E^{*} a}{2 R}+\frac{E^{* 2} \pi a^{3}}{16 R^{2}}-2 E^{*} \Delta \gamma=0,
$$

to determine the contact half width $a$ as a function of the external pulling force $F$ and the pulling angle $\theta$.

The explicit solution of Eq. (23) can be written as

$$
F=-\frac{\pi E^{*} a^{2} \sin \theta}{4 R}+\sqrt{2 \pi E^{*} \Delta \gamma a-\frac{\pi^{2} E^{* 2} a^{4} \cos ^{2} \theta}{16 R^{2}} .}
$$

In order to obtain the pull-off force, we take

$$
\frac{\partial F}{\partial a}=0,
$$

which yields the pull-off contact half width $a_{\mathrm{p}}$ as

$\frac{a_{\mathrm{p}}}{R}=\left(\frac{8 \Delta \gamma\left[1+\sin ^{2} \theta-\sqrt{\left.\left(1+\sin ^{2} \theta\right)^{2}-\cos ^{2} \theta\right]}\right.}{\pi E^{*} R \cos ^{2} \theta}\right)^{1 / 3}$.

Inserting the pull-off contact half width $a_{\mathrm{p}}$ in Eq. (26) into Eq. (24) leads to the pull-off force $F_{\mathrm{p}}$ in a direction of $\theta$,

$$
\frac{F_{\mathrm{p}}}{\Delta \gamma}=\frac{-\pi E^{*} a_{\mathrm{p}}^{2} \sin \theta}{4 \Delta \gamma R}+\sqrt{\frac{2 \pi E^{*} a_{\mathrm{p}}}{\Delta \gamma}-\frac{\pi^{2} E^{* 2} a_{\mathrm{p}}^{4} \cos ^{2} \theta}{16 R^{2} \Delta \gamma^{2}}} .
$$

As a special case, $\theta=\pi / 2$, the relation between the pulling force and the contact half width becomes

$$
F=\frac{-\pi E^{*} a^{2}}{4 R}+\sqrt{2 \pi E^{*} a \Delta \gamma},
$$

the pull-off contact half width in this case is

$\frac{a_{\mathrm{p}}}{R}=\left(\frac{2 \Delta \gamma}{\pi E^{*} R}\right)^{1 / 3}$

and the pull-off force is

$$
\frac{F_{\mathrm{p}}}{\Delta \gamma}=\frac{-\pi E^{*} a_{\mathrm{p}}^{2}}{4 R \Delta \gamma}+\sqrt{\frac{2 \pi E^{*} a_{\mathrm{p}}}{\Delta \gamma}}
$$

which are identical to the plane strain classical JKR theory [8].

Using the pull-off force of JKR theory, $F_{\mathrm{pJKR}}$, in Eq. (30), to normalize that of nonoscillatory solution to the present model yields

$$
\frac{F_{\mathrm{p}}}{F_{\mathrm{pJKR}}}=-\frac{4}{3}\left(\frac{\omega}{2}\right)^{2 / 3} \sin \theta+\frac{2^{4 / 3}}{3} \sqrt{4 \omega^{1 / 3}-\omega^{4 / 3} \cos ^{2} \theta}
$$

where

$$
\omega=\frac{1+\sin ^{2} \theta-\sqrt{\left(1+\sin ^{2} \theta\right)^{2}-\cos ^{2} \theta}}{\cos ^{2} \theta} .
$$

The corresponding normalized pull-off contact half width is

$$
\frac{a_{\mathrm{p}}}{a_{\mathrm{pJKR}}}=\left(\frac{4\left[1+\sin ^{2} \theta-\sqrt{\left(1+\sin ^{2} \theta\right)^{2}-\cos ^{2} \theta}\right]}{\cos ^{2} \theta}\right)^{1 / 3},
$$

$a_{\mathrm{pJKR}}$ is identical to $a_{\mathrm{p}}$ in Eq. (29).

From Eqs. (31) and (33), one can see that the normalized pull-off force ad pull-off contact half width are only dependent 
on the pulling angle $\theta$ and have no relations with the pulling force and the materials' constants.

If we define the adhesive strength as

$\sigma=\frac{F_{\mathrm{p}}}{2 a_{\mathrm{p}}}$

then, the normalized adhesive strength can be expressed as

$\frac{\sigma}{\sigma_{\mathrm{JKR}}}=\frac{F_{\mathrm{p}}}{F_{\mathrm{pJKR}}} \frac{a_{\mathrm{pJKR}}}{a_{\mathrm{p}}}$,

where $\sigma_{\mathrm{JKR}}$ is the adhesive strength in the classical JKR theory.

\section{Discussions}

\subsection{The effects of Dundurs' parameter $\beta$}

As mentioned above, we have recently studied three kinds of generalized cases with perfect bonding [35-37]: (1) generalized JKR model of an elastic cylinder adhesively contacting with an elastic stretched substrate [35]; (2) generalized MaugisDugdale model of an elastic cylinder adhesively contacting with an elastic stretched substrate [36]; (3) generalized JKR model with perfectly adhesive bonding between two dissimilar elastic spheres [37]. In all these three cases, it has been found that the effects of Dundurs' parameter $\beta$ on the contact half width (contact radius) and the pull-off process are negligible and a nonoscillatory solution can be a good approximation to the generalized solution for each model. In all these three cases, the external pulling force $F$ vanishes or the pulling direction is normal to the contact interface, which is the main difference from the present model.

In this section, the effects of Dundurs' parameter $\beta$ will also be discussed with consideration of different pulling angle in the present model.

According to Eq. (16), Fig. 2 denotes the pulling force $F$ normalized by that of nonoscillatory solution $(\beta=0)$ at the normalized contact half width $a / R=0.1$ versus different Dundurs' parameter $\beta$, in which two values of $\Delta \gamma / E^{*} R$ are taken as 0.001 and 0.01 , respectively, and three representative pulling

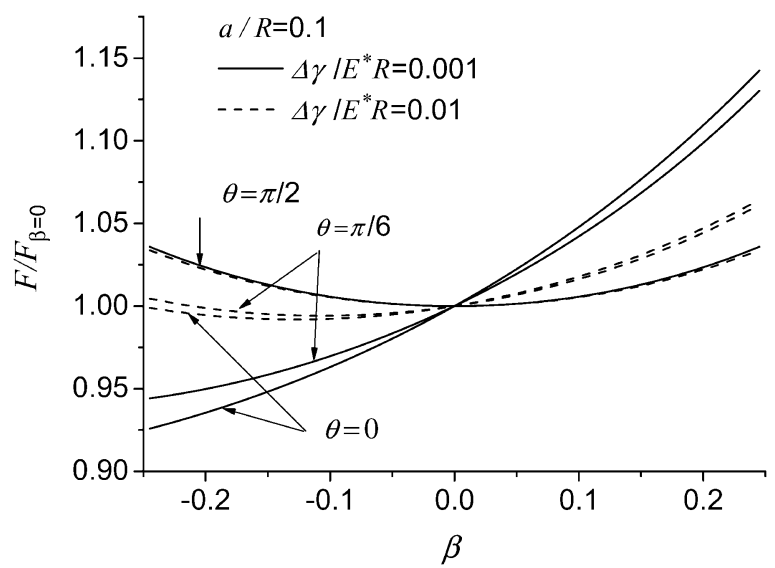

Fig. 2. Plots of normalized pulling force $F / F_{\beta=0}$ versus Dundurs' parameter $\beta$ with the normalized contact half width $a / R=0.1$, parameters $\Delta \gamma / E^{*} R=$ $0.001,0.01$ and the pulling angles $\theta=0, \pi / 6, \pi / 2$, to check the effects of $\beta$. angles are investigated, $\theta=0, \pi / 6, \pi / 2$. The region of $\beta$ is taken from -0.25 to 0.25 , the admissible values for most materials. From Fig. 2, one can see that the difference between the oscillatory solution and nonoscillatory one is influenced by the nondimensional parameter $\Delta \gamma / E^{*} R$ and the pulling angle $\theta$. The smaller the pulling angle $\theta$ and nondimensional parameter $\Delta \gamma / E^{*} R$, the larger difference it has.

Fig. 3 gives the relation between the normalized pulling force $F / F_{\beta=0}$ at the normalized contact half width $a / R=0.05$ and the Dundurs' parameter $\beta$. The other parameters are taken the same as those in Fig. 2. Comparing Figs. 2 and 3, one can see that for a fixed bimaterial pair and a fixed pulling angle, the smaller that contact width, the smaller the difference between the oscillatory and nonoscillatory solutions is.

In Fig. 4, normalized pulling force $F / F_{\beta=0}$ at the normalized contact half width $a / R=0.1$ via the pulling angle $\theta$ for two different nondimensional parameters $\Delta \gamma / E^{*} R$ is given, in which three typical values of Dundurs' parameter $\beta$ are chosen, i.e., $\beta=-0.25,0,0.25$. From Fig. 4 , one can see that effects of Dundurs' parameter decrease when the nondimen-

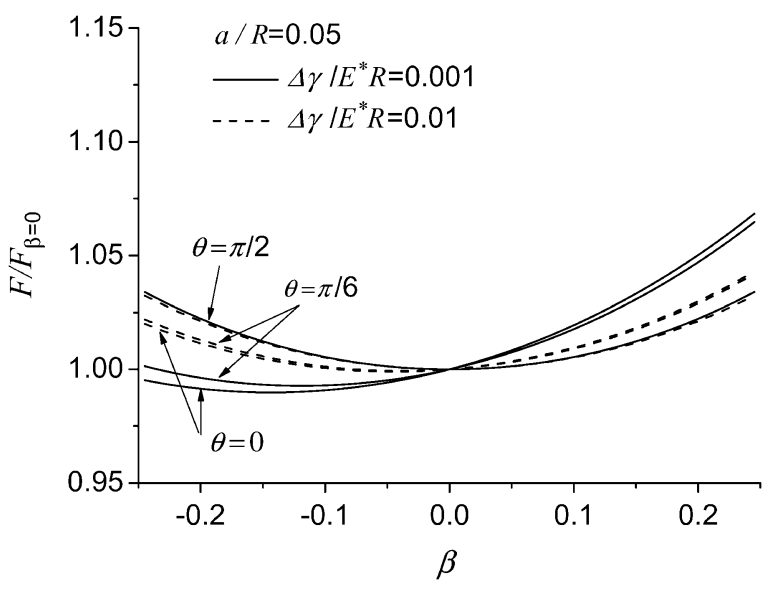

Fig. 3. Plots of normalized pulling force $F / F_{\beta}=0$ versus Dundurs' parameter $\beta$ with the normalized contact half width $a / R=0.05$, parameters $\Delta \gamma / E^{*} R=$ $0.001,0.01$ and the pulling angles $\theta=0, \pi / 6, \pi / 2$, to check the effects of $\beta$.

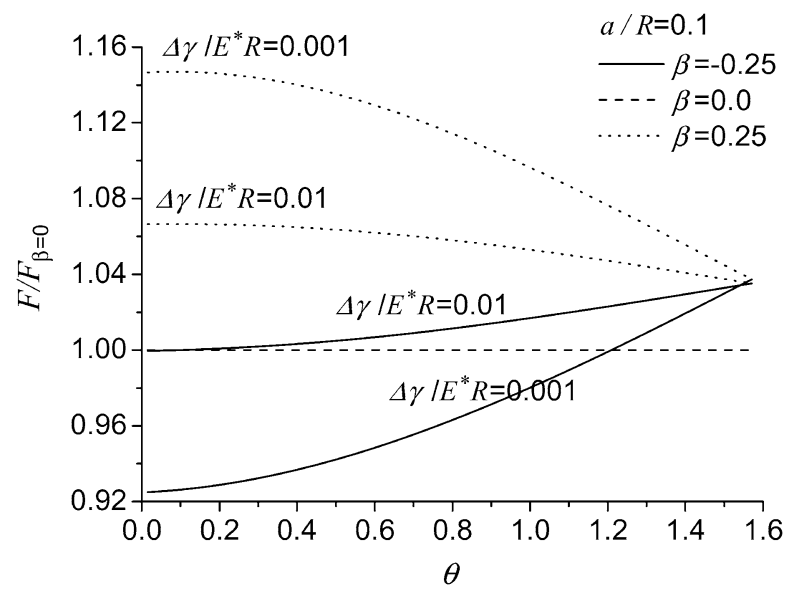

Fig. 4. Curves of normalized pulling force $F / F_{\beta=0}$ versus pulling angle $\theta$ with the normalized contact half width $a / R=0.1$, parameters $\Delta \gamma / E^{*} R=0.001$, 0.01 and three typical Dundurs parameters $\beta=-0.25,0,0.25$ to check the difference between the general solution and the nonoscillatory one. 
sional parameter $\Delta \gamma / E^{*} R$ increases. The maximum influence of Dundurs' parameter on the pull-off process happens in the case with pulling angle $\theta=0$.

Considering a different normalized contact half width, for example, $a / R=0.05$, and the other parameters are taken the same as those used in Fig. 4, the relations between the normalized pulling force $F / F_{\beta=0}$ and pulling angle $\theta$ are shown in Fig. 5. Comparing Figs. 4 and 5, we can find that the effects of Dundurs' parameter on the pull-off process decreases as the contact half width decreases. As the value of nondimensional parameter $\Delta \gamma / E^{*} R$ is larger than 0.001 , the effects of Dundurs' parameter in any case is negligible. The difference between the oscillatory solution and the nonoscillatory one is less than $7 \%$.

In a whole, the effects of Dundurs' parameter is not significant for larger pulling angle, smaller contact half width or larger nondimensional parameter $\Delta \gamma / E^{*} R$. Under these conditions, the nonoscillatory solution can generally be a good approximation for the model of an elastic cylinder adhesively contacting with an elastic half space with a pulling force on the cylinder in an arbitrary direction.

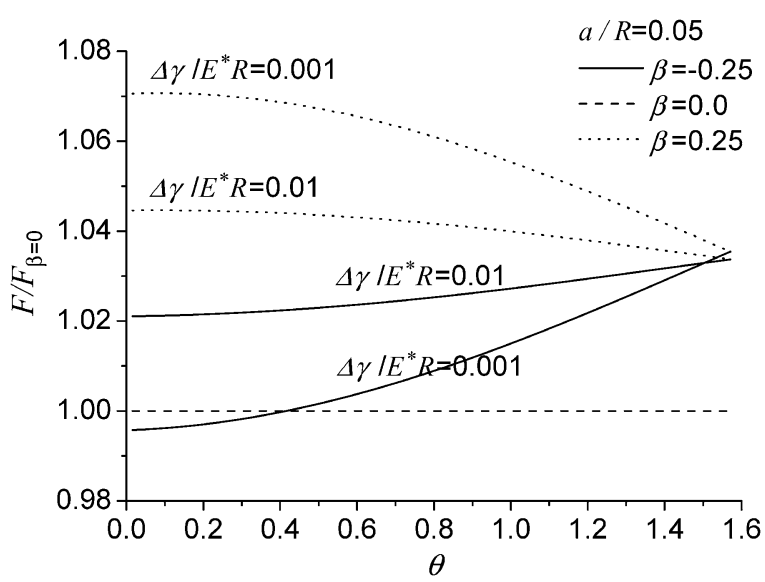

Fig. 5. Curves of normalized pulling force $F / F_{\beta}=0$ versus pulling angle $\theta$ with the normalized contact half width $a / R=0.05$, parameters $\Delta \gamma / E^{*} R=0.001$, 0.01 and three typical Dundurs parameters $\beta=-0.25,0,0.25$ to check the difference between the general solution and the nonoscillatory one.

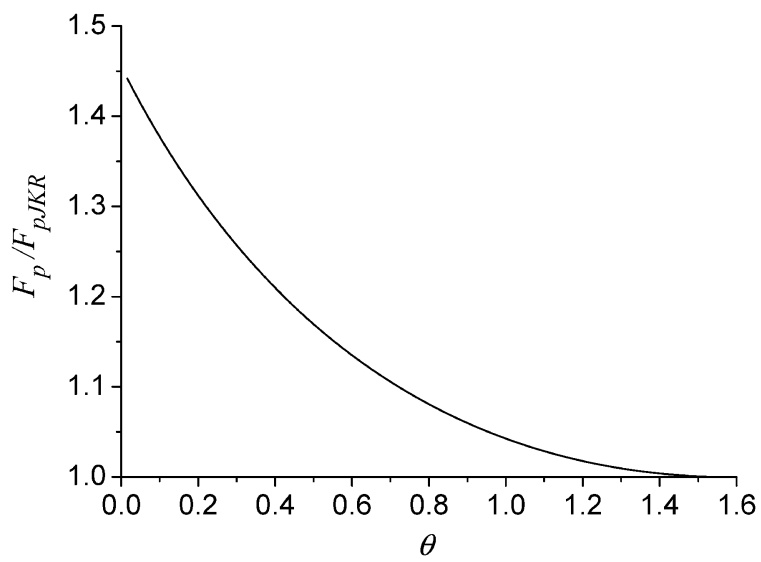

Fig. 6. The effects of pulling angle $\theta$ on the pull-off force $F_{\mathrm{p}}$, where $F_{\mathrm{pJKR}}$ is the pull-off force in the classical JKR theory. The maximum pull-off force emerges at $\theta=0$.

\subsection{The effects of the pulling angle on the pull-off process}

In order to get some understanding on how the pulling angle influencing the pull-off force and pull-off contact half width in the approximate solutions, Eqs. (31), (33), and (35) are analyzed numerically here. Fig. 6 denotes the normalized pull-off force $F_{\mathrm{p}} / F_{\mathrm{pJKR}}$ versus the pulling angle $\theta$. From Eqs. (31) and (32), we know that for the nonoscillatory solution, the normalized term $F_{\mathrm{p}} / F_{\mathrm{pJKR}}$ has no relation with the material constants and pulling force, only depends on the pulling angle $\theta$. It shows that the pull-off force decreases when the pulling angle increases and attains the maximum value at $\theta=0$.

Fig. 7 gives a curve of the relation between the normalized pull-off contact half width $a_{\mathrm{p}} / a_{\mathrm{p} \text { JKR }}$ and the pulling angle $\theta$, which shows that the pull-off contact half width decreases as the pulling angle increases and attains a maximum value at $\theta=0$. In Fig. 8, the adhesive strength $\sigma / \sigma_{\mathrm{JKR}}$ versus the pulling angle $\theta$ is plotted according to Eq. (35). From Fig. 8, one can see that the adhesive strength increases as the pulling angle increases and obtains a maximum value at $\theta=\pi / 2$.

From the above discussion, one should note that the nonoscillatory solution give a good approximation to the bimaterial

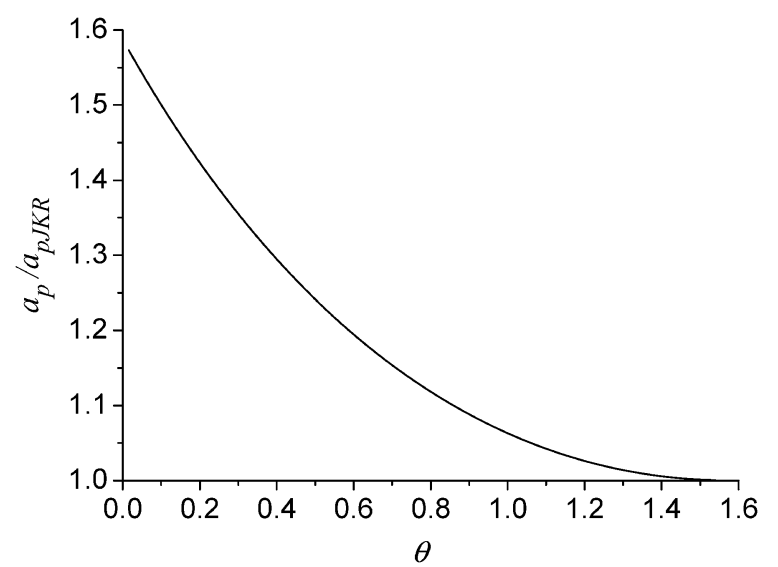

Fig. 7. The effects of pulling angle $\theta$ on the pull-off contact half width $a_{\mathrm{p}}$, where $a_{\mathrm{pJKR}}$ is the pull-off contact half width in the classical JKR theory. The maximum pull-off contact half width is at $\theta=0$.

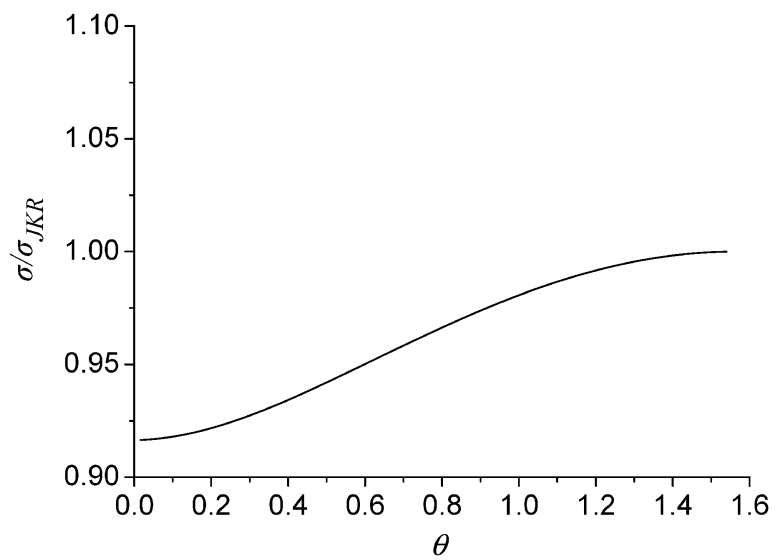

Fig. 8. The effects of pulling angle $\theta$ on the adhesive strength $\sigma$, where $\sigma_{\mathrm{JKR}}$ is the adhesive strength in the classical JKR theory. The maximum adhesive strength is at $\theta=\pi / 2$. 


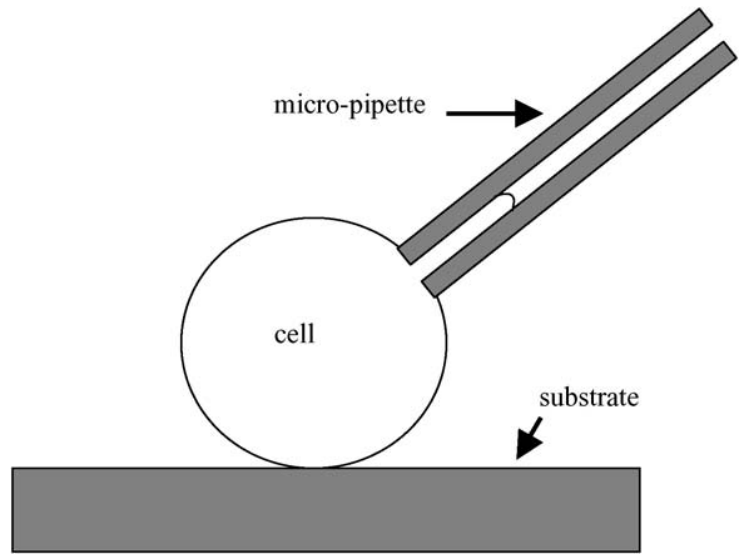

Fig. 9. Scheme of a micro-pipette to measure the adhesive force of a cell cultured on a substrate.

model under some conditions. Otherwise, the exact oscillatory one should be used for analysis or applications. As a special case of the present model, $\theta=\pi / 2$ [35-37] have demonstrated that nonoscillatory solution is a good approximation, which can also be found in the present model.

Solutions to the contact model between orthotropic or anisotropic bodies will be investigated in the future studies as well as viscoelasticity features.

As we know that [24] has used the classical JKR mode to successfully predict the adhesion energy between two S180 cells. The present model is promising to predict the adhesive force between cells and substrates using micro-pipette aspiration as schemed in Fig. 9, which is also a usual way used in the bio-experiments.

\section{Acknowledgments}

The work reported here is supported by NSFC (No. 10202023) and SC would like to express his gratitude to Professor $\mathrm{H}$. Gao (working in Brown University now) for his help when SC visited Max-Plank Institute for Metals Research, Stuttgart, Germany, during 2003 and 2005.

\section{References}

[1] H. Hertz, J. Reine Angew. Math. 92 (1882) 156.

[2] K.L. Johnson, K. Kendall, A.D. Roberts, Proc. R. Soc. London A 324 (1971) 301.
[3] B.V. Derjaguin, V.M. Muller, Y.P. Toporov, J. Colloid Interface Sci. 53 (1975) 314

[4] D. Tabor, J. Colloid Interface Sci. 58 (1976) 1.

[5] D. Maugis, J. Colloid Interface Sci. 150 (1992) 243.

[6] V.M. Muller, V.S. Yushenko, B.V. Derjaguin, J. Colloid Interface Sci. 77 (1980) 91

[7] J.A. Greenwood, K.L. Johnson, Philos. Mag. 43 (1981) 697.

[8] M. Barquins, J. Adhes. 26 (1988) 1.

[9] R.W. Carpick, N. Agrait, D.F. Ogletree, M. Salmeron, Langmuir 12 (1996) 3334.

[10] M.K. Chaudhury, T. Weaver, C.Y. Hui, E.J. Kramer, J. Appl. Phys. 80 (1996) 30

[11] K.L. Johnson, J.A. Greenwood, J. Colloid Interface Sci. 192 (1997) 326

[12] J.M. Baney, C.Y. Hui, J. Adhes. Sci. Technol. 11 (1997) 393.

[13] J.A. Greenwood, Proc. R. Soc. London A 453 (1997) 1277.

[14] E. Barthel, J. Colloid Interface Sci. 200 (1998) 7.

[15] F. Robbe-Valloire, M. Barquins, Int. J. Adhes. Adhes. 18 (1998) 29.

[16] J.A. Greenwood, K.L. Johnson, J. Phys. D Appl. Phys. 31 (1998) 3279.

[17] K.S. Kim, R.M. McMeeking, K.L. Johnson, J. Mech. Phys. Solids 46 (1998) 243.

[18] C. Morrow, M. Lovell, X. Ning, J. Phys. D Appl. Phys. 36 (2003) 534.

[19] U.D. Schwarz, J. Colloid Interface Sci. 261 (2003) 99.

[20] O.T. Sari, G.G. Adams, S. Muftu, J. Appl. Mech. 72 (2005) 633.

[21] K.L. Johnson, Contact Mechanics, Cambridge Univ. Press, Cambridge, UK, 1985.

[22] K. Kendall, J. Phys. D Appl. Phys. 8 (1975) 1722.

[23] A.R. Savkoor, G.A.D. Briggs, Proc. R. Soc. London A 356 (1977) 103.

[24] Y.S. Chu, S. Dufour, J.P. Thiery, E. Perez, F. Pincet, Phys. Rev. Lett. 94 (2005) 028102.

[25] K. Autumn, M. Sitti, Y.C. Liang, A.M. Peattie, W.R. Hansen, S. Sponberg, T.W. Kenny, R. Fearing, J.N. Israelachvili, R.J. Full, Proc. Natl. Acad. Sci. USA 99 (2002) 12252.

[26] E. Artz, S. Gorb, R. Spolenak, Proc. Natl. Acad. Sci. USA 100 (2003) 10603.

[27] B.N.J. Persson, J. Chem. Phys. 118 (2003) 7614.

[28] A.K. Geim, S.V. Dubonos, I.V. Grigorieva, K.S. Novoselov, A.A. Zhukov, S. Shapoval, Nature Mater. 2 (2003) 461.

[29] H. Gao, H. Yao, Proc. Natl. Acad. Sci. USA 101 (2004) 7851.

[30] N.J. Glassmaker, A. Jagota, C.Y. Hui, J. Kim, J. R. Soc. Interface 1 (2004) 23.

[31] C.Y. Hui, N.J. Glassmaker, T. Tang, A. Jagota, J. R. Soc. Interface 1 (2004) 35.

[32] H. Yao, H. Gao, J. Mech. Phys. Solids 54 (2006) 1120.

[33] H. Gao, X. Wang, H. Yao, S. Gorb, E. Arzt, Mech. Mater. 37 (2005) 275.

[34] R. Spolenak, S. Gorb, H. Gao, E. Artz, Proc. R. Soc. London A 461 (2005) 305.

[35] S. Chen, H. Gao, Proc. R. Soc. London A 462 (2006) 211.

[36] S. Chen, H. Gao, Int. J. Mater. Res. (formy Zeitschrift für Metallkunde) 97 (2006) 584.

[37] S. Chen, H. Gao, J. Mech. Phys. Solids 54 (2006) 1548.

[38] J. Dundurs, J. Appl. Mech. 36 (1969) 650. 